\title{
Advances in High-Resolution Microscale Impedance Sensors
}

\author{
Marco Carminati \\ Dipartimento di Elettronica, Informazione e Bioingegneria, Politecnico di Milano, 20133 Milano, Italy \\ Correspondence should be addressed to Marco Carminati; marcol.carminati@polimi.it
}

Received 5 September 2016; Revised 30 January 2017; Accepted 15 February 2017; Published 12 March 2017

Academic Editor: Chenzhong Li

Copyright (C) 2017 Marco Carminati. This is an open access article distributed under the Creative Commons Attribution License, which permits unrestricted use, distribution, and reproduction in any medium, provided the original work is properly cited.

\begin{abstract}
Sensors based on impedance transduction have been well consolidated in the industry for decades. Today, the downscaling of the size of sensing elements to micrometric and submicrometric dimensions is enabled by the diffusion of lithographic processes and fostered by the convergence of complementary disciplines such as microelectronics, photonics, biology, electrochemistry, and material science, all focusing on energy and information manipulation at the micro- and nanoscale. Although such a miniaturization trend is pivotal in supporting the pervasiveness of sensors (in the context of mass deployment paradigms such as smart city, home and body monitoring networks, and Internet of Things), it also presents new challenges for the detection electronics, reaching the zeptoFarad domain. In this tutorial review, a selection of examples is illustrated with the purpose of distilling key indications and guidelines for the design of high-resolution impedance readout circuits and sensors. The applications span from biological cells to inertial and ultrasonic MEMS sensors, environmental monitoring, and integrated photonics.
\end{abstract}

\section{Introduction}

Impedance, being the frequency-dependent ratio between the two fundamental electrical parameters, voltage and current, is an ubiquitous quantity, widely leveraged to realize sensors at different size scales. A physical quantity becomes detectable by means of impedance (i.e., converted into a measurable electrical signal) when a variation of such a quantity reflects into a variation of the impedance of the sensor (Figure 1). Typically, impedance sensors are passive: energy is not directly transferred from the physical domain to the electrical domain (as in the case of optical and radiation sensors, where photons are converted into electrons, or of electrochemical sensors, where charge is transferred from ions to metal electrodes during a redox reaction). Here, external events change the sensor impedance and a stimulation source is required to inject some energy into the sensor (either a voltage or a current) in order to detect such a variation.

For decades, impedance sensors have been well consolidated in the industry thanks to their versatility and to two key properties: they are (1) noninvasive and (2) contactless. The former attribute is granted by the adjustable penetration of the electric field in the matter, while the latter is due to charge induction (capacitive coupling).
The interface between the physical domain (i.e., the sensing material/device) and the electrical domain is the electrodes. Although the design of the electrodes is often overlooked, the codesign of the electrodes and the readout electronics is a key point to achieve optimal performance, as illustrated in the following. Thanks to the advancements and consolidation of microfabrication technologies, particularly of photolithography, now accessible also to medium- and small-scale cleanrooms and not only to large microelectronics foundries, today the size of the electrodes can be shrunk down to the micrometric and submicrometric scale. Beyond compactness, such miniaturization enables a brand new range of applications, since micron-sized objects become directly and individually detectable by means of impedance. In particular, that is the case of micrometric solid particles, micromachined silicon moving structures, and biological cells.

If, on the one hand, miniaturization enables unprecedented spatiotemporal resolution and paves the way to a broad range of pervasive biomicrosensing applications, on the other hand, it makes the measurement of impedance more challenging. There are two main difficulties: (1) the signal scales down with the sensor size, thus requiring lower noise to detect impedance variations $\Delta Z$, and (2) the connection parasitics between the sensor and the readout electronics, 


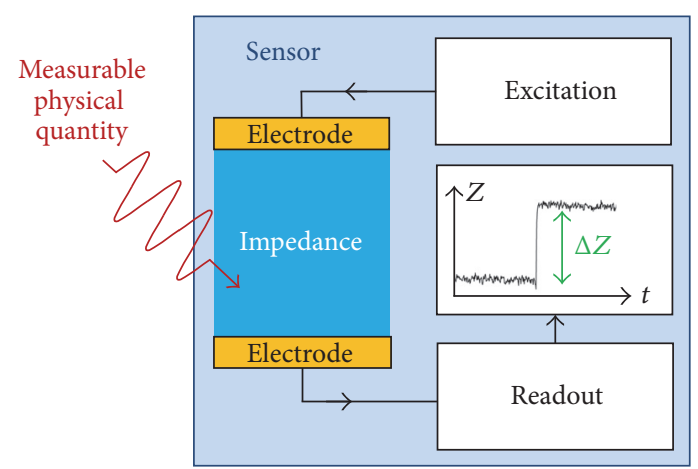

FIGURE 1: Architecture of an impedance sensor composed of a stimulation and a readout circuit. The resolution is the minimum impedance variation $\Delta Z$ that can be detected.

often, do not scale, thus becoming the dominant term to be minimized. In this tutorial review, selected case studies are presented in order to highlight valuable solutions and design criteria to address these detection challenges.

\section{Classification, Applications, and Performance}

Despite the great variety of impedance sensors, they can be grouped into a few classes according to the sensing principle. The major classification is between resistive and capacitive sensors. Other grouping criteria are the detection principle (material versus geometry change), the size of the sensor, the arrangement (single versus differential), the achievable resolution (smallest $\Delta Z$ in Figure 1), the speed of response, the dynamic range (DR), the probing frequency $f_{\mathrm{AC}}$, and the type of readout circuit.

2.1. Resistive Sensors. Resistive sensors measure the change of resistance, the real part of impedance. Resistance depends on the material resistivity $\rho$ and the geometry. Thus, a variation of resistance can be due to a change of the resistivity or of the sensor geometry (as in the case of resistive strain gauge). Neglecting border effects, for a parallel plate geometry (with facing electrodes of area $A$, separated by a distance $D), R,=\rho D / A$, while for other geometries a cell constant $k$ is defined so that $R=\rho k$. For a coplanar geometry, the resistance and capacitance between a pair of parallel band electrodes can be calculated by means of conformal mapping [1], providing analytical expressions for $k$.

The resistivity depends on the number of charge carriers in the material and on their mobility. Thus, modulation of resistivity can be due to a change in the number of charge carriers (electrons in metals and semiconductors, ions in electrolytic solutions) such as in the case of photoconductors, or to a change of the mobility. The latter is due, for instance, to temperature variations, as in the case of RTD (resistive temperature detectors) such as the very popular Pt100 (made of platinum, characterized by a positive temperature coefficient), or to the effect of the magnetic field as in magnetoresistive sensors $[2,3]$.

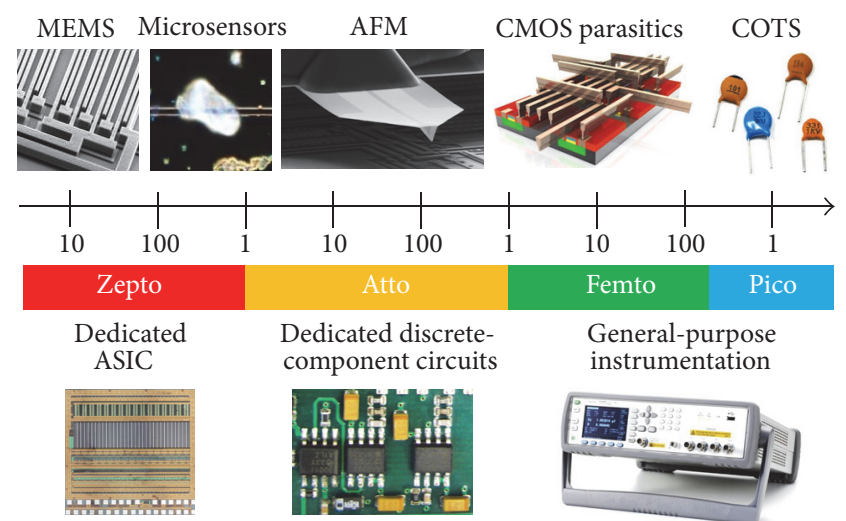

FIGURE 2: Ranges of capacitive resolution achievable by means of different instrumentation and setup solutions.

Very interestingly, it must be noted that resistance can be measured, also, in a contactless way, when in series to the resistance $R$ there is a capacitance $C$. Such a capacitance can be physically present in the device (such as in the case of the double-layer capacitance of ionic resistors and the CLIPP sensor illustrated in the following section) or placed externally, as a physical electronic component (capacitor) in the detection circuit.

2.2. Capacitive Sensors. Analogously to resistive sensors, capacitive ones measure the change in capacitance between two electrodes separated by a dielectric material. Such variation can be due to a change of the material (specifically of its dielectric constant $\varepsilon_{r}$ ) or of the geometry. Two classical applications are the measurement of linear displacement and the measurement of liquid level in a reservoir. In the former case, the dielectric is constant (typically air) and the geometry changes since one electrode is fixed and the other one moves. In the latter case, the electrodes are fixed, while the level of liquid (with $\varepsilon_{r}$ higher than air) changes the capacitance. Analogously, different liquids flowing in channels can be identified by the different $\varepsilon_{r}[4]$ and debris can be detected [5].

When multiple electrodes are employed, capacitance and impedance tomography can be performed, for instance, placing an array of electrodes around organs in the body (for noninvasive biomedical imaging and artificial skin sensing [6]) or around an insulating pipe to track the liquid composition [7]. Three-dimensional tomographic maps can be reconstructed with proper algorithms solving the inverse problem, by sequentially stimulating one electrode and measuring the response of all the others.

The evolution of industrial capacitive sensors clearly shows the constant miniaturization trend and the corresponding need for increasingly better resolution. Figure 2 illustrates the relation between capacitive resolution and the type of instrumentation correspondingly needed. A resolution in the femtoFarad $\left(10^{-15} \mathrm{~F}\right)$ range, suitable, for instance, to measure the parasitic coupling between CMOS metal lines, is provided by commercial instrumentation. High resolution, in the attoFarad $\left(10^{-18} \mathrm{~F}\right)$ range, is required for 
particular applications, such as nanometric displacement [8] or local impedance sensing with the conductive tip of the Atomic Force Microscope (AFM) [9] and is achievable with dedicated electronics realized with discrete components [911]. Ultrahigh resolution, in the zeptoFarad $\left(10^{-21} \mathrm{~F}\right)$ range, is only achievable with a monolithic combination of the microelectrodes with the ASIC implementing the readout circuits $[12,13]$. A quantitative analysis of the capacitive resolutions achievable in different applications is reported in the survey of Table 1, where the sensors are ordered for decreasing size. $C$ is the total sensor capacitance, $\Delta C$ is the typical variation of capacitance, $\sigma_{C}$ is the sensor resolution, $\mathrm{DR}$ is the dynamic range ( $\mathrm{DR}=C / \sigma_{\mathrm{C}}$ ), and SNR is the signalto-noise ratio $\left(\Delta C / \sigma_{C}\right)$. Also, the table shows whether the sensing method (Meas.) is based on material or geometry variation $(M / G)$, whether the implementation (Impl.) is monolithic or based on discrete components $(M / D)$, and whether a differential configuration is adopted. However, in the millimeter size range, touchscreen capacitive detection is inserted in the table, representing the top bound with $\mathrm{pF}$ range capacitances, currently being a mainstream application in industry [14]. Another interesting industrial application, despite being less recent and commercially successful, is fingerprint recognition by means of capacitive sensors. In the reported case, the sensor is monolithic, realized in CMOS technology, with an array of 16 '380 microelectrodes $(30 \mu \mathrm{m}$ $\times 30 \mu \mathrm{m}$ ) fabricated in the top metal layer and buried under $2 \mu \mathrm{m}$ thick dielectric passivation [15]. Evolutions of liquid level sensing in the microfluidics domain include level gauging in a microreservoir with pL sensitivity [16] and detection of droplets [1]. Manipulation of single water droplets in air or, more commonly, in oil is performed in the context of digital microfluidics, where the droplets are individually actuated by means of passivated planar electrodes, leveraging the phenomenon of electrowetting on dielectrics (EWOD). In the majority of cases, electrodes are in $\mathrm{mm}$ size range and the corresponding $C$ is in the $\mathrm{pF}$ range, while a more scaled solution has been proposed [17].

\section{Microscale Case Studies}

3.1. Cells. As mentioned in the Introduction, the most interesting biological entities of micrometric size that can be probed by impedance are cells, whose size ranges from 15 to $20 \mu \mathrm{m}$ of mammalian cells down to $1-2 \mu \mathrm{m}$ of bacteria. The simplest electrical equivalent of electrically passive cells is a single shell (Figure 3(a)) composed by a membrane capacitance (of specific surface capacitance $C_{M} \sim 0.01 \mathrm{pF} / \mu \mathrm{m}^{2}$ ) associated with the cell insulating membrane (a bilipidic layer of $4 \mathrm{~nm}$ thickness) in series to the impedance of the cytoplasm $\left(R_{C} \| C_{C}\right)$, very similar to the culture medium, which is a high-conductivity (typically $1.5 \mathrm{~S} / \mathrm{m}$ ) water-based solution (with approximately the same dielectric constant of water). Consequently, the presence of the cell can be electrically detected thanks to the contrast between the conductive saline medium and the insulating cell body [18]. Typically, it can be considered insulating for frequencies up to the $\mathrm{MHz}$ range, above which the membrane capacitance is bypassed.

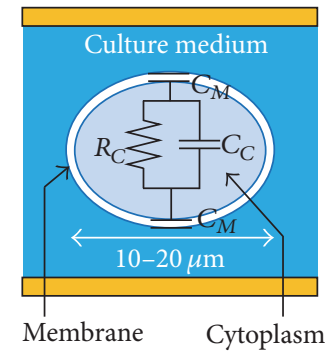

(a) Cell impedance model

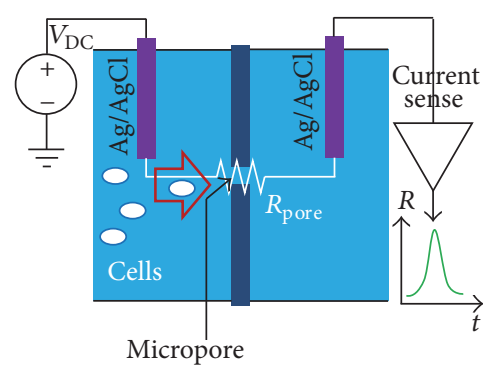

(b) Coulter counter principle
FIGURE 3: (a) Electrical equivalent of a nonelectrogenic cell; (b) scheme of the orifice-based Coulter sensing approach.

Traditionally, single mammalian cells have been electrically counted and sized by means of the Coulter counter [19]. In this case, the micrometric bodies to be detected are forced to pass through a small aperture (slightly wider than the largest cell) separating two large chambers filled with ionic solution, contacted with macroscopic $\mathrm{Ag} / \mathrm{AgCl}$ electrodes providing ohmic contact with the liquid (Figure 3(b)). The passage of a single cell temporarily obstructs the micropore, producing an increase of the measured ionic resistance between the two chambers. The width of the resistive pulse depends on the translocation speed (transit time typically in the order of $100 \mu \mathrm{s}$ ) and the amplitude depends on the volume of the cells, thus allowing extracting the size of the cells. The resolution is typically in the \% range. Thanks to the ohmic contact of nonpolarizable electrodes, the ionic resistance is measured in DC, allowing great simplicity in the readout circuit. This technique proved to be very powerful and has been extended from cells, reducing the size of the pore down to nanometric dimensions, also to detect nanoparticles at high speed [20], DNA molecules (for label-free sequencing) [21], and bacteria [22] and clusters of antibodies in pharmaceutical plants [23].

The key design aspect of the resistive pulse detection is that, in order to increase the sensitivity, the diameter of the pore should be as small as possible, matching the size of the target. As an example, the same readout electronics can detect single bacteria $(1-2 \mu \mathrm{m}$ ) with a large pore of $3 \mu \mathrm{m}$ by $5 \mu \mathrm{m}$ (and $50 \mu \mathrm{m}$ long) [24], as well as single nanoparticles of $220 \mathrm{~nm}$ diameter with an orifice cross section of $1.4 \mu \mathrm{m}$ by $2 \mu \mathrm{m}$ (and $5 \mu \mathrm{m}$ long) [25]. Unfortunately, this implies two significant drawbacks: (i) high risk of device clogging, if debris or larger clusters of particles/cells happen to flow through the aperture, and (ii) reduced flow rate. Higher flow rates can be achieved by putting in parallel several channels and by replicating the readout electronics. Another critical point is that the dynamic range is limited, since the signal scales with the volume of the particle. In order to extend the dynamic range, a channel with nonconstant cross section has been proposed: "nodes" along the channel produce a size-specific signal (signature) that, by means of autocorrelation, enables improving the SNR [26].

When targeting biomolecules (such as proteins, antibodies, enzymes, or other biomarkers) of nanometric size, that is, much smaller than single cells, two approaches can be 


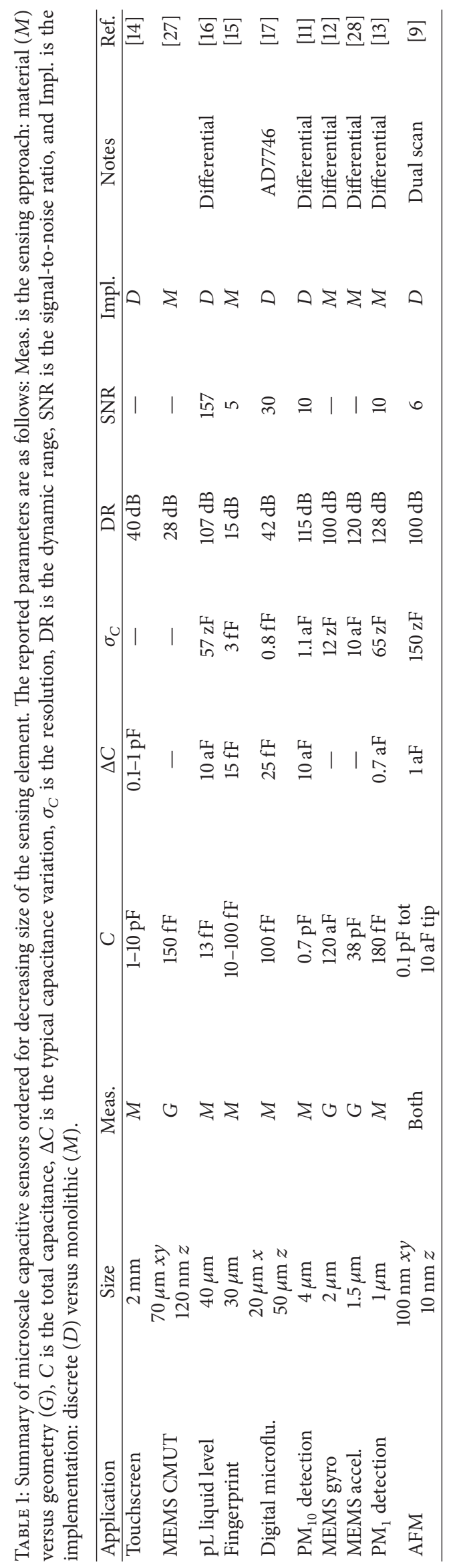




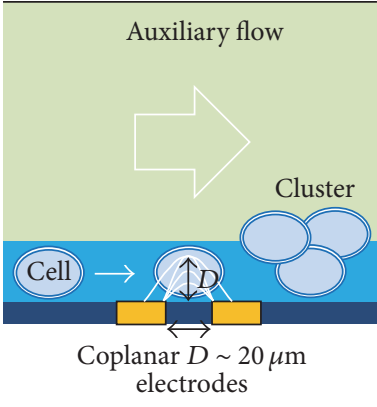

(a) Flow cytometry

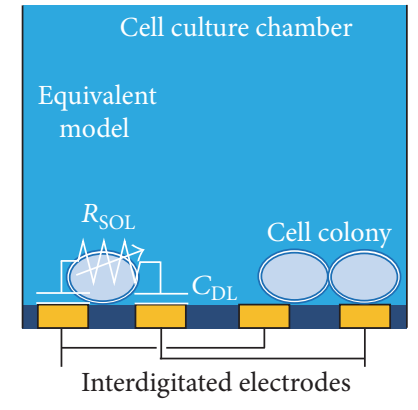

(b) Growth monitoring
FIGURE 4: Use of impedance for detecting cells (a) flowing in a single stream in a microfluidic channel and (b) growing on a planar substrate.

followed. One way is to fabricate nanometric orifices and optimize the performance of the detection electronics, for instance, by minimizing the stray capacitance, thus preferring glass nanopipettes to silicon nanopores [29, 30]. Another approach is to functionalize larger beads with target-specific receptors that cause agglomeration of beads in the presence of the target molecules. Recent examples include the use of $7 \mu \mathrm{m}$ polystyrene beads [31], $2.8 \mu \mathrm{m}$ magnetic beads [32], and $290 \mathrm{~nm}$ nanoparticles [33] that, combined with an $800 \mathrm{~nm}$ pore, allowed single-molecule resolution. Magnetic beads functionalized with specific receptors allow also the use of an external magnetic force to differentiate among cells expressing specific molecules, for instance, by using two micropores in series and slowing [34] or capturing [35] them and comparing the peaks before and after the interaction with the magnetic field.

An alternative to the micropore approach, significantly less prone to the clogging risk, is the use of microelectrodes for the confinement of the electric field. In particular, if we consider a pair of coplanar band microelectrodes separated by a distance $D$ (Figure $4(\mathrm{a})$ ), the electric field protrudes in the vertical direction for a distance equal to about $D$. Thus, if the distance $D$ is made comparable to the cell size $(10-20 \mu \mathrm{m})$, the passage of a single cell in this sensing volume (of crosssectional area $\sim D^{2}$ ) significantly perturbs the impedance between the two electrodes. Clearly, the cells must flow very close to the surface where the microelectrodes are fabricated. However, the channel can be much wider, thus compatible with the transit of larger objects, without any risk of clogging, provided that a focusing mechanism is used to vertically align the stream of cells close to the sensing area. Hydrodynamic focusing is typically the preferred choice, being suitable for three-dimensional focusing and independent of the particle/cells properties. It requires only additional pumps to drive the auxiliary inlets for the sheath flows [36]. This technique is called impedance flow cytometry (IFC) [37]: analogously to the Coulter counter, it enables counting and sizing cells since the variation of impedance is related to the cell volume [38]. Furthermore, if different cell types express different electrical phenotypes (e.g., live versus dead cells), sorting based on impedance is also possible [39], as a label-free alternative to the standard optical approach (FACS, Fluorescence-Activated Cell Sorting), enabling high throughput $\left(10^{4} \mathrm{cells} / \mathrm{sec}\right)$ and potential for molecular sensitivity but requiring staining cells with fluorophores.

Beyond the absence of the orifice, the most important difference between the two approaches is in the electrode/solution interface. The microelectrodes used in flow cytometry are fabricated by means of lithography in noble metals such as gold or platinum, which do not easily exchange charge with the ionic solution. Thus, the interface is polarizable and a double-layer capacitance $\left(C_{\mathrm{DL}}\right.$ in Figure $\left.4(\mathrm{~b})\right)$ needs to be bypassed at $f_{\mathrm{AC}}$ in order to access the solution resistance $\left(R_{\mathrm{SOL}}\right)$ modulated by the presence of the cells. For the common case of gold/cell culture buffer, the value of $C_{\mathrm{DL}}$ per unit area of the electrodes is $\sim 0.1 \mathrm{pF} / \mu \mathrm{m}^{2}$.

Along with the dynamic use of impedance in flow cytometry, it is also well suitable for static monitoring of the growth of a colony of cells, adherent on a solid substrate. In this case (Figure 4(b)), the layer of growing cells that progressively cover the substrate alters the interfacial impedance. The impedance increase can be detected by means of a single large electrode, as in the pioneering technique called ECIS (Electric Cell-substrate Impedance Sensing) [40] or by means of interdigitated electrodes with a distance comparable with the cells size [41]. The latter proved to be more sensitive thanks to the confinement of the electric field better matched with the volume of cells [42]. After decades, this technique is well consolidated and three commercial systems are available in the market: ECIS $Z$ by Applied Biophysics $\left(f_{\mathrm{AC}} 4-64 \mathrm{kHz}\right)$ and Cell Key by Molecular Devices $\left(f_{\mathrm{AC}} 1 \mathrm{kHz}-10 \mathrm{MHz}\right)$ have a single disk electrode, while exCELLigence by Roche $\left(f_{\mathrm{AC}} 10-50 \mathrm{kHz}\right)$ is based on interdigitated electrodes. Since when cells die they commonly detach from the substrate and impedance decreases, the death of the colony can be tracked as well [43]. The full life cycle of cells, from seeding to death, can be recorded. This is a key aspect, which makes this technique perfectly suited for high-throughput drug discovery since the response of cells to the perfusion of new drugs can be screened in automatic, parallelizable, and combinatorial ways. The only limitation of this technique is the strong dependence of the signal on the adhesion of cells, depending on the surface properties (chemical and morphological) and on the specific cell line. Thus, quantitative impedance results cannot be easily migrated from one cell type to another. Reliable results can be obtained only by comparing impedance values for the same cell type.

Despite the vast literature (reviewed in [37] up to 2010), single-cell IFC has not yet reached the same maturity and diffusion of ECIS. From the analysis of recent advances in the field of single-cell detection [44-59], the following trends can be highlighted. (1) Smarter and more robust event detection algorithms are emerging. Instead of simply fixing a threshold on the impedance value exceeded by the resistive pulse for each passage, digital segmentation and autocorrelation leveraging the odd symmetry of differential pulses can be used [44], as well as multielectrode structures that, while increasing the input capacitance and the measurement time, produce specific signatures, whose shape can be better identified against noise [45] or improved electrode 
layout [46]. (2) Combination of IFC with dielectrophoresis (DEP) is increasing for sorting [47], trapping [48], orienting [49], and sensing [50]. Beyond hydrodynamic focusing, the uncertainty due to the spread in the vertical position of cells can be reduced by creating a vertical gradient in the value of $C_{\mathrm{DL}}$ of vertical electrodes [51]. (3) Beyond counting and sizing, significantly richer information can be extracted from impedance: morphology of single cells can be dynamically screened [49] combining simultaneous measurements among orthogonal arrangements of electrodes, as well as with an array of electrodes for static single-cell microtomography, for instance, to study electroporation [52]. In order to analyze subcellular entities, a higher probing frequency needs to be used; in particular, dielectric spectroscopy (e.g., at $1 \mathrm{GHz}$ across $30 \mu \mathrm{m}$ spaced electrodes in a resonator measured in a bulky microwave setup [53]) is becoming a very interesting technique, complementary to standard IFC, commonly limited below $100 \mathrm{MHz}$. (4) Focus is increasing on single-cell analysis leveraging fast trapping of single cells by means of DEP [48] or microbarriers, for instance, of cross section $10 \mu \mathrm{m} \times 5 \mu \mathrm{m}$ [54] demonstrating the capability to discriminate ex vivo young versus old vascular endothelial cells showing a difference of $12 \mu \mathrm{s}$ in $R_{C}$ and of $0.7 \mathrm{pF}$ in $C_{M}$. Impedance detection is increasingly combined with actuators that are triggered by the translocation of a single cell. In fact, single cells can be "printed" in droplets of $500 \mathrm{pL}$ volume by combining IFC with piezoactuation in real-time loop [55] and can be imaged by a CMOS image sensor, directly coupled to a microfluidic channel and triggered by a differential resistive pulse sensor [56]. When several channels are put in parallel to increase the total flow rate, replicating the impedance AC readout might be an issue at discrete-component level, and thus a combination of several electrodes into a single port preserving single-cell resolution by means of spatially coded orthogonal sequences has been recently proposed [57].

Finally, it must be highlighted that, also in the context of ECIS, single-cell resolution represents the current state of the art, achieved, for instance, by using ISFETs with a micrometric gate area matched with the size of single cells, that is, $2 \mu \mathrm{m} \times 16 \mu \mathrm{m}$ [58] or $5 \mu \mathrm{m} \times 12 \mu \mathrm{m}$ [59].

3.2. CLIPP. A recent and original application of impedance in the field of integrated photonics consists in the measurement of the conductance of a semiconductor waveguide for inferring the intensity of light propagating within it. Although the semiconductor is transparent at the operational wavelength (typically $1550 \mathrm{~nm}$ ) since the energy gap of the semiconductor is larger than the energy of the photons, a very small fraction of light is absorbed due to intermediate energy states, lying in the gap and created by the presence of lattice defects at the interface between the core and the cladding. By measuring the tiny increase of photogenerated carriers (i.e., of conductance), it is possible to measure the local light intensity. A direct ohmic contact with the waveguide is not feasible, since it would heavily attenuate the propagating electromagnetic field. The simple solution adopted in the CLIPP (ContactLess Integrated Photonic Probe) is to capacitively access the waveguide (Figure 5(a)) by placing two electrodes at a safe distance $(\sim 1 \mu \mathrm{m})$ on the top cladding [60]. Thus, (a)

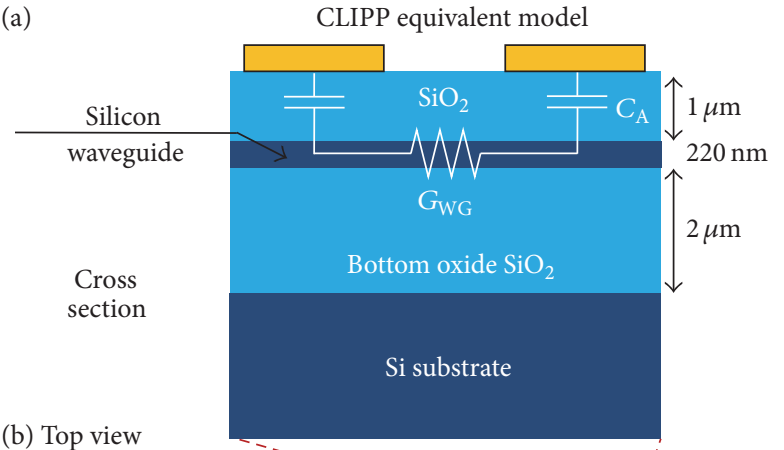

(b) Top view

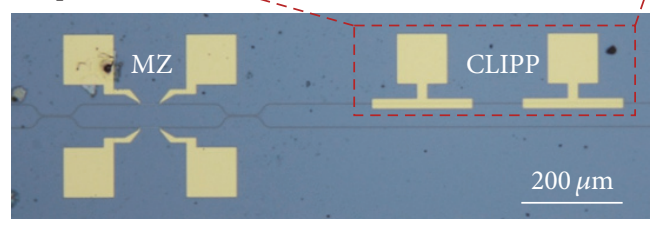

(c)

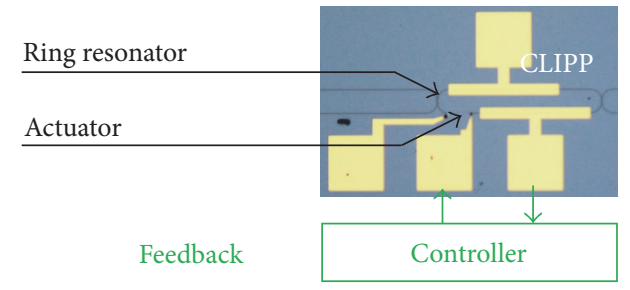

FIGURE 5: CLIPP longitudinal cross section (a) highlighting the simplified impedance model and top view of two devices: (b) MZ interferometer switch and (c) ring resonator with embedded CLIPPs allowing closed-loop stabilization and control.

the CLIPP is compatible with all fabrication technologies, requiring no additional postprocessing or modifications to the design of the optical layer which is decoupled from the sensing layer. Such a top metal layer is a commonly available fabrication step, typically used to pattern thermooptical actuators for tuning the devices.

The simplified sensor equivalent impedance is composed of two access capacitances $\left(C_{A}\right.$ in Figure 5(a)) between the electrodes and the waveguide, through the top cladding $(1 \mu \mathrm{m}$ thick $\mathrm{SiO}_{2}$ for SOI technology where the waveguide has a thickness of $220 \mathrm{~nm}$ and a width of $480 \mathrm{~nm}$ for single-mode operation), in series to the waveguide conductance $\left(G_{\mathrm{WG}}\right)$. The impedance probing frequency should be chosen high enough to bypass $C_{A}$ and measure $G_{\mathrm{WG}}$. If the bottom oxide is sufficiently thick $(>2 \mu \mathrm{m})$, the capacitive coupling toward the silicon substrate manifests at slightly higher frequencies, delimiting a resistive plateau.

The CLIPP operation has been demonstrated on both silicon [60] and InP [61] photonic platforms. For silicon, the CLIPP offers $-35 \mathrm{dBm}$ sensitivity, $40 \mathrm{~dB}$ dynamic range in the $1.3-1.6 \mu \mathrm{m}$ spectrum, and a response time of tens of $\mu \mathrm{s}$, with a minimum linear footprint in the order of $100 \mu \mathrm{m}$. Since no additional loss is introduced, several CLIPPs can be cascaded along the same optical path, making this solution suitable for optical circuits at large scale of integration. Versatile placing of the CLIPP inside each optical device and massive parallelization are finally enabled. For optimal 
performance and multichannel readout, up to 32 CLIPPs can be operated with a single custom-designed low-noise CMOS ASIC tracking the sensors impedance [62].

Since the CLIPP provides local information of light intensity, it can be used inside a feedback loop, in combination with an actuator, to tune and control the operation of a photonic device. Examples of devices highly sensitive to fabrication tolerances and temperature drift are MachZehnder interferometers used as switches (Figure 5(b)) [63] and microring resonators (Figure 5(c)) [64] largely used in filters and multiplexers, both successfully stabilized by embedded CLIPP sensors.

The most promising competitor technology to the CLIPP for realizing embedded light power monitors and closedloop control is still based on surface state absorption [65], requiring more complex and invasive fabrication of reversebiased lateral PN junctions, used to collect the photogenerated carriers.

If closed-loop control can solve the issue of thermal sensitivity of optical components [66], their scalability is hampered by a power dissipation issue [67]. Considering that the power dissipation of single thermal actuator is currently in the order of tens of mW, packing hundreds or thousands of them on a single chip poses severe energetic issues. Thus, alternative technologies for light switches are being explored, which do not require static dissipation such as MEMS with electrostatic actuation [68] or ionic intercalation [69].

3.3. MEMS. The area of MEMS (Microelectromechanical Systems) was among the first to deploy impedance measurement at the microscale. Despite the great variety of technologies (hybrid versus monolithic integration, bulk versus surface machining) and devices, we review here two kinds of sensors where high-resolution capacitive measurement of displacement is fundamental: inertial sensors and CMUT (Capacitive Micromachined Ultrasonic Transducers). In both cases, capacitance is used to detect the motion between a fixed electrode and a moving one. In the case of inertial sensors, both accelerometers [28] and gyroscopes [12], a proof mass, anchored to the fixed frame through silicon springs, moves under the effect of inertial forces. The capacitance is measured between two combs of interdigitated electrodes, one fixed to the frame and the other moving with the seismic mass (Figure 6(a)). A fully differential geometry is adapted since, with respect to the balanced rest position, when moving in one direction, the capacitance increases on one side and decreases on the opposite side. In a monolithic implementation, a chip by Analog Devices (ADXRS family of gyros) features a resolution of $12 \mathrm{zF}\left(V_{\mathrm{AC}}=10 \mathrm{~V}, \mathrm{BW}=0.1 \mathrm{~Hz}\right.$, and $\left.f_{\mathrm{AC}}=15 \mathrm{kHz}\right)[12]$.

CMUT devices, largely used, for instance, in biomedical imaging [70], transduce ultrasound waves into electrical signal by means of the flexure of a thin $(\sim 200 \mathrm{~nm})$ micromachined membrane. As illustrated in Figure 6(b), the membrane can be postfabricated on top of CMOS chip where arrays of sensing pads $(70 \mu \mathrm{m} \times 70 \mu \mathrm{m})$ measure the capacitance with respect to thin electrodes embedded in the membrane at a distance of $120 \mathrm{~nm}$ [27].

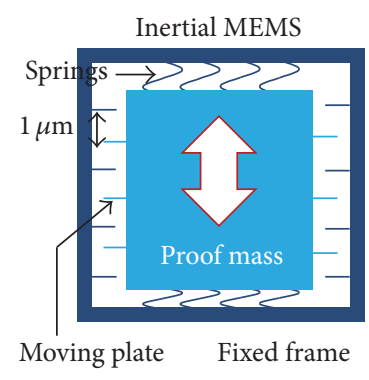

(a)

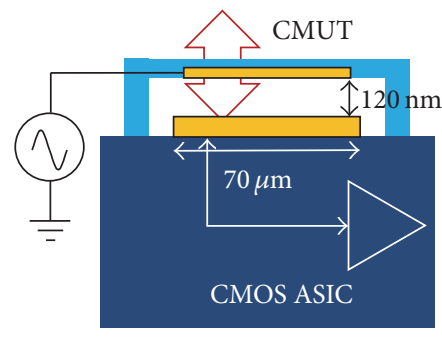

(b)
FIGURE 6: High-resolution capacitive sensing in MEMS: (a) inertial sensors and (b) ultrasonic transducers.

Beyond the application to silicon micromachined devices, high-resolution capacitive sensing is leveraged also in combination with piezoactuators in order to achieve a closed loop with nanometric resolution [8].

3.4. Particulate Matter. Starting from the basic consideration that the largest airborne particulate matter $\left(\mathrm{PM}_{10}\right.$, with an equivalent diameter below $10 \mu \mathrm{m}$ ) has the same size of biological cells, it was proposed to adopt an electric approach also to detect single dust particles in air [11]. The fundamental difference is that for cells in liquid there is a high contrast (about $10^{6}: 1$ ) in conductivity between the saline solution and the insulating cells. Instead, for particles in air, the electrical difference is in terms of dielectric constant, which can be as small as $2: 1$, with $\varepsilon_{r}$ of plastic being just double that of air. Another complication is related to the transport of the particles: since air has a density 1000 times smaller than water, offering no buoyancy, it is more difficult to guide them through microchannels. Consequently, instead of an in-flow architecture, bottom planar electrodes are used, on which passive or forced deposition of PM is capacitively detected.

Single large $\mathrm{PM}_{10}$ particles have been capacitively detected with gold microelectrodes patterned on a glass substrate and connected to a low-noise readout circuit achieving $1 \mathrm{aF}$ resolution. Since the coplanar electrodes had a minimum distance of $4 \mu \mathrm{m}$, the corresponding capacitive signal is in the $10 \mathrm{aF}$ range, giving a minimum detectable diameter of about $7 \mu \mathrm{m}$.

In order to target $\mathrm{PM}_{1}$, whose concentration is more relevant from the toxicological point of view, a fully monolithic approach was adopted. As illustrated in Figure 7, the interdigitated electrodes have been fabricated with the top metal layer of CMOS chip ( $0.35 \mu \mathrm{m}$ AMS process) exposed to air with the same windowing used for bonding pads [13]. Beyond miniaturization and potential for mass production, this monolithic solution provides two key advantages: smaller electrodes can be fabricated (in this case the width and separation are equal to $1 \mu \mathrm{m}$ ) and the readout electronics are realized on the same chip, achieving radical minimization of the connection parasitics and, correspondingly, optimal resolution (as illustrated in the next section).

The electrodes are interdigitated and connected in a differential configuration. In order to cover a sensing area 


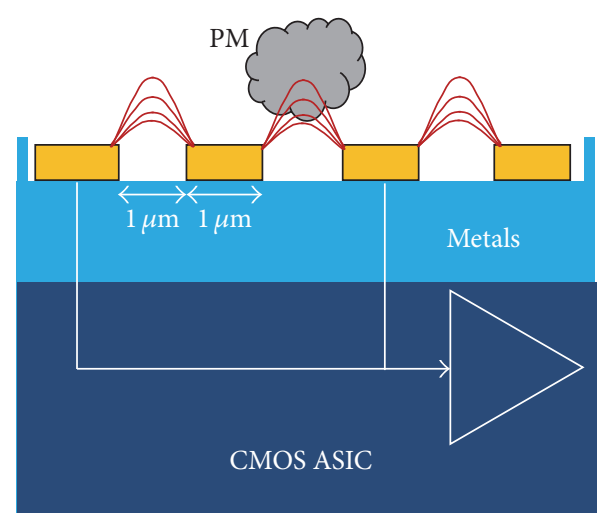

(a)

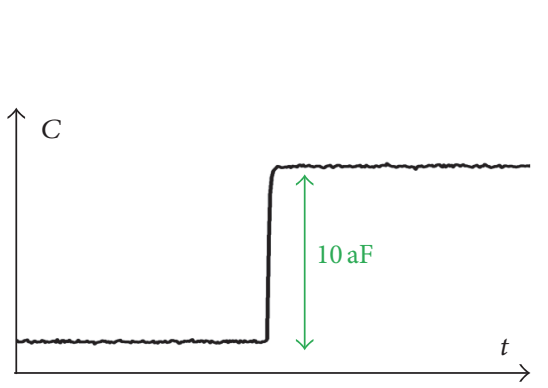

(b)

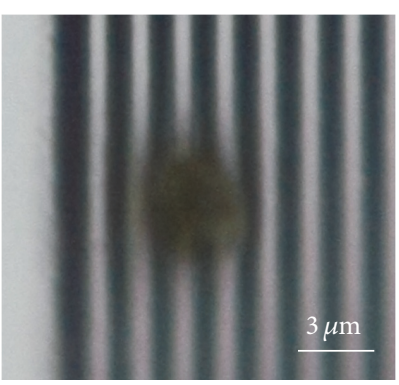

(c)

FIGURE 7: On-chip detection of single dust particles of $1 \mu \mathrm{m}$ diameter with monolithic CMOS sensors (a) featuring a resolution of $65 \mathrm{zF}$ (b) and $1 \mu \mathrm{m}$ interdigitated electrodes (c) on an active deposition area of $1 \mathrm{~mm}^{2}$.

of $1 \mathrm{~mm}^{2}, 32$ channels $(500 \mu \mathrm{m} \times 70 \mu \mathrm{m})$ are connected in parallel. Each channel features a low-noise front-end with capacitive feedback, an automatic network for compensating the mismatch between electrodes pairs (up to $4.5 \mathrm{fF}$ with 5bit resolution), a square-wave multiplier, and $g_{m}-C$ low-pass filter. The capacitive resolution is $65 \mathrm{zF}$ with a bandwidth of $40 \mathrm{~Hz}$, corresponding to a dynamic range of $128 \mathrm{~dB}$ (at $3.3 \mathrm{~V}$ power supply, $3.3 \mathrm{~V}$ square-wave forcing signal, and $25 \mathrm{~mA}$ current consumption). This resolution allows detecting particles of diameter down to $1 \mu \mathrm{m}$ : if made of carbon (i.e., with the lowest $\varepsilon_{r}=2$ ), it produces $\Delta C \sim 0.7 \mathrm{aF}$, clearly detectable with $\mathrm{SNR} \sim 20 \mathrm{~dB}$. Figure 7 (b) shows the measured capacitive jump due to the landing of a single mineral talc particle of $\sim 3.5 \mu \mathrm{m}$ diameter (visible on top of the electrodes in the microscope image of Figure $7(\mathrm{c}))$ producing a step $\Delta C=10 \mathrm{aF}$ (with an excellent SNR in excess of $40 \mathrm{~dB}$ ) [13].

The major drawbacks of the capacitive approach (need for an active particle capturing force to reduce the measurement time, need for periodic cleaning of the detection surface, and sensitivity to false counts due to humidity droplets) are shared with the other solid-state competitor technology based on mass-sensitive silicon resonators [60, 61], whose resonance frequency shifts according to the increase of mass due to PM deposition, currently targeting smaller diameters [71] or lacking single-particle resolution in the $\mathrm{PM}_{10}$ range [72].

\section{Design Criteria}

From the analysis of the selected case studies, briefly discussed above and summarized in Table 2, we can synthetize useful indications and design guidelines for the optimization of high-resolution impedance sensors.

4.1. Low-Noise Readout Electronics. Of course, the design of the readout circuit is pivotal in achieving high resolution. An important consideration is that the electronics should be designed together with the electrodes [73]. If some degrees of freedom are available also on the electrodes side, the codesign phase should be iterative, adapting the electrodes to
TABLE 2: Summary of the presented microscale case studies.

\begin{tabular}{|c|c|c|}
\hline Type & Material & Geometry \\
\hline $\mathrm{DC}$ & $\begin{array}{l}\text { RTD, magnetoresistance } \\
\text { Coulter counter }\end{array}$ & Strain gauge \\
\hline $\mathrm{AC}$ & $\begin{array}{c}\text { Flow cytometry } \\
\text { CLIPP }\end{array}$ & \\
\hline C & $\begin{array}{l}\text { Particulate matter } \\
\text { Liquid level, droplets } \\
\text { fingerprint, touch }\end{array}$ & $\begin{array}{c}\text { MEMS inertial sensors } \\
\text { MEMS CMUT } \\
\text { Piezo-nano-displacement }\end{array}$ \\
\hline
\end{tabular}

the minimization of noise and tailoring the electronics on the electrodes impedance.

Impedance can be measured in several ways, for instance, putting the unknown $Z$ inside a bridge composed of adjustable impedances, or coupling it to the time constant of a resonator or oscillator, in order to convert the signal into a frequency shift. For ultralow-noise performance, the optimal readout scheme, adopted in all the case studies presented above, is composed of a current reading front-end combined with a narrowband phase-sensitive detector (the lock-in detector). As illustrated in Figure $8, Z$ is excited with a voltage signal $V_{\mathrm{AC}}$ (typically a sinusoidal signal at frequency $f_{\mathrm{AC}}$ ) and the current correspondingly flowing in $Z$ is read by a transimpedance amplifier (TIA), converting it, through the feedback impedance, into a voltage $V_{\text {OUT }}$, fed to the lock-in demodulator (LID). The LID consists of a multiplier, shifting the signal from $f_{\mathrm{AC}}$ to $\mathrm{DC}$ and, by a low-pass filter (LPF) with a tunable bandwidth (BW), removing the replica of the spectrum around $2 f_{\mathrm{AC}}$ and setting the sensor response time. Being synchronized with the excitation signal $V_{\mathrm{AC}}$, the LID can extract the in-phase $(\operatorname{Re}[Z])$ and in-quadrature $(\operatorname{Im}[Z])$ components of $Z$. The advantages of this scheme with respect to other solutions are as follows:

(1) Precise control of the voltage $V_{A C}$ applied across $Z$ thanks to the virtual ground. This is particularly relevant for electrochemical and biological applications where cells and macromolecules are very sensitive to the applied field and potential. 


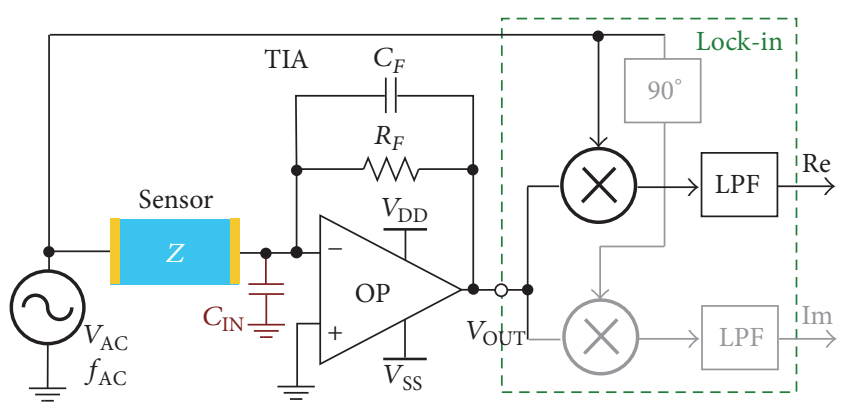

FIgURE 8: Low-noise impedance detection circuit combining current-sensing with synchronous lock-in demodulation.

(2) Neutralization of parasitics since to whole sensor current is absorbed by the virtual ground (when the TIA loop gain is high) and no current flows in the parasitic paths between the terminals of $Z$ and ground.

(3) Tunability of BW independently of $f_{A C}$ : a narrow BW can be set $(\sim \mathrm{Hz})$ around large $f_{\mathrm{AC}}(\sim \mathrm{MHz})$, achieving in a simple way an equivalent band-pass filter with very high quality factor $\left(f_{\mathrm{AC}} / \mathrm{BW} \sim 10^{7}\right)$ and automatically tracking a changing $f_{\mathrm{AC}}$, that is, ideal for impedance spectroscopy.

(4) Minimum noise, thanks to the narrow filter around $f_{\mathrm{AC}}$.

(5) Compatibility with both monochromatic (single frequency at $\left.f_{\mathrm{AC}}\right)$ and nonmonochromatic stimulation waveforms (multisine, square waves, wide spectrum such as pseudo noise [74], etc.).

(6) Compatibility with single-terminal sensing (i.e., when the second terminal is grounded and not accessible by the readout circuit) by exciting the virtual ground instead of the $Z$ terminal, as illustrated in Figure 9(a). It requires subtraction of the $V_{\mathrm{AC}}$ signal at $V_{\mathrm{OUT}}$ node.

(7) Compatibility with both single-ended and differential schemes by simply adding an inverting buffer (Figure 9(b)).

(8) Versatility: the implementation of the LID can be either analog (by means of analog multipliers [75]) or digital (PC-based [10] or FPGA-based [76]).

The TIA, despite being very effective in reading the sensor current with a low input impedance, which neutralizes other parasitics, presents a severe limitation: it is affected by a fundamental resolution/speed trade-off. Since the only design parameter is the value of the feedback resistor $R_{F}$, opposite trends collide in its sizing. For achieving high transimpedance gain and low thermal noise (current power spectral density equal to $4 \mathrm{kT} / R_{F}$, where $k$ is the Boltzmann constant and $T$ is the absolute temperature), $R_{F}$ should be maximized. On the other hand, due to the intrinsic parasitic capacitance (minimum $C_{F}=0.2 \mathrm{pF}$ for any SMD component), the amplifier bandwidth is $1 /\left(2 \pi R_{F} C_{F}\right)$, thus requiring smaller $R_{F}$ to obtain wide bandwidth. Advanced

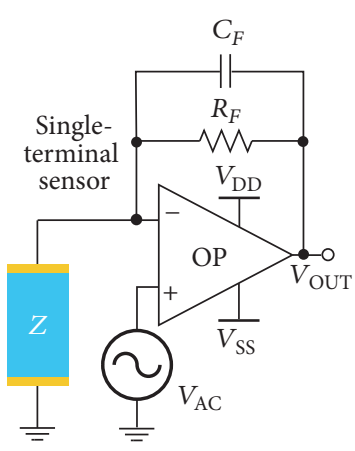

(a)

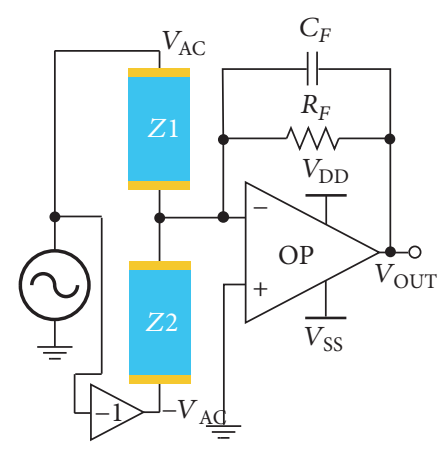

(b)
FIgURE 9: (a) Single-side impedance measurement and (b) differential configuration.

topologies can be adopted to relax this trade-off, for instance, by removing $R_{F}$, thus operating the first stage as low-noise integrator (ideal for capacitive sensors) complemented with networks for handling DC leakage currents and additional stages such as differentiators to extend the bandwidth by cancelling the integrator slow pole [77].

The performances of advanced analog front-end topologies for capacitive sensing are summarized in Table 3, including both CMOS integrated [78-81] and discrete-component implementations. Since the resolution scales linearly with $V_{\mathrm{AC}}$ and worsens with the square root of $\mathrm{BW}$, a valid figure of merit (FoM) for the comparison of the ultimate resolution offered by different circuits is $\mathrm{FoM}=\sigma_{\mathrm{C}} \cdot V_{\mathrm{AC}} / \sqrt{ } \mathrm{BW}$, that is, the noise spectral density normalized on the stimulation amplitude, as reported in the table. The best performing circuits in the zeptoFarad range are all based on ASIC monolithic operation.

Power dissipation, unavoidably large for low-noise circuits, is also reported as a secondary parameter. In fact, its importance strongly depends on the application. Clearly, power dissipation is of primary importance for batteryoperated sensors, while it can be traded off in exchange for low noise in high-resolution applications.

4.2. Parasitics and Miniaturization. The ultimate resolution limit is set by the front-end noise, whose contribution should be minimized (ideally below other intrinsic sources of noise). A key aspect for the minimization of the input current noise of the TIA front-end is the minimization of the total capacitance at the virtual ground node, $C_{\mathrm{IN}}$ in Figure 8. In fact, the input-referred equivalent voltage noise source of $\mathrm{OP}$ causes fluctuations of the virtual ground potential which are differentiated across $C_{\mathrm{IN}}$, producing a noise current. Due to the differentiation, this noise term linearly increases with the frequency and with the value of $C_{\mathrm{IN}}$ (see a detailed noise analysis in [77]). This is the fundamental reason why the minimization of the length of the connection between the electrodes and the amplifier pad is so important, leading to the radical solution of making the experiment/sensor "on the pad," that is, building the sensing microelectrodes on the same ASIC substrate, as shown for MEMS gyros [12], CMUT [27], dust detection [13], on-chip capacitive biosensors [83], 


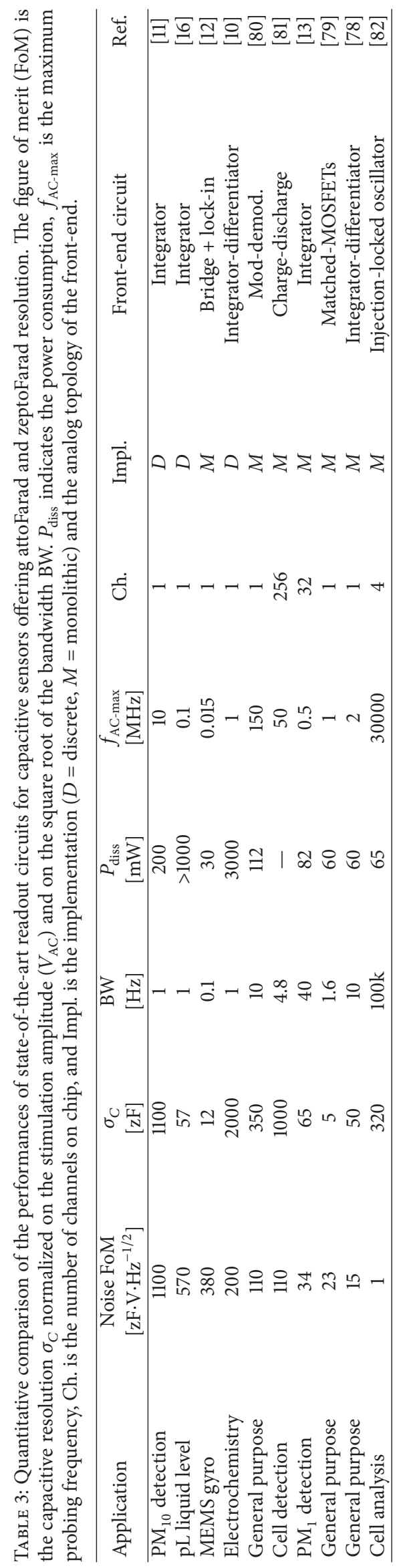


and nanopore current amplifier [84] and AFM impedance spectroscopy on pad [85].

In order to properly minimize $C_{\mathrm{IN}}$, it is important to extract an accurate model of the total device impedance, including parasitic terms. For devices realized by means of lithography, the substrate plays a fundamental role. If the ASIC is also realized on the same substrate, it must be clearly a silicon substrate, and the distance from the electrodes to the substrate should be maximized (as in the case of the CLIPP and $\mathrm{PM}_{1}$ detector). Instead, if the substrate is passive, that is, a purely mechanical support of microelectrodes as in electrochemical cartridge chips coupled to microfluidics [41], then an insulating substrate such as glass or quartz should be definitely preferred [86].

4.3. Frequency and Signal Optimization. Along with the minimization of noise, it is of course important to maximize the signal. Thus, rather than focusing only on $\sigma_{C}$, the most important parameter to optimize is the SNR that must be maximized. A simple but clarifying example is the pore-based Coulter scheme for detection of insulating microparticles in a conductive solution (Figure 3(a)). Since the power spectral density of the thermal current noise of the pore ionic resistance $R_{\text {pore }}$ is $4 \mathrm{kT} / R_{\text {pore }}$, in order to reduce the device noise, $R_{\text {pore }}$ should be increased by reducing the conductivity $\sigma_{\mathrm{SOL}}$ of the solution. However, the signal is proportional to $\sigma_{\mathrm{SOL}}\left(\Delta I \sim \sigma_{\mathrm{SOL}}\right.$, since the larger the value of $\sigma$, the larger the contrast with an insulating object). Since the SNR $\sim \sigma_{\mathrm{SOL}} / \sqrt{ } \sigma_{\mathrm{SOL}}$, that is, the signal linearly increases with $\sigma_{\mathrm{SOL}}$, while the noise increases only with the square root of $\sigma_{\mathrm{SOL}}$, in the end, it is better to increase $\sigma_{\mathrm{SOL}}$.

To achieve signal maximization when measuring a resistance $R$ in series to a capacitance $C$ (as in the CLIPP case), the probing frequency $f_{\mathrm{AC}}$ should be carefully chosen. In the absence of a tight upper limit to the resistive plateau, $f_{\mathrm{AC}}$ should be at least one decade higher than $1 /(2 \pi R C)$, in order to avoid the signal drop across the capacitor impedance. Unfortunately, a variety of factors often limit the upper end of the plateau. On the one hand, the impact of all the stray capacitances becomes more important for increasing frequency, since more current correspondingly flows in the parasitic paths. For example, in the case of the CLIPP sensor, the stray capacitive coupling to the conductive silicon substrate severely limits the extension of the plateau. On the other hand, operating at high frequency is more demanding for the electronics (wide-band amplifiers and components operating at higher frequencies) and the impact of delays and phase errors, for instance, due to long cables or improper PCB layout, again becomes critical. If possible, in order to avoid these complications, the size of the sensor should be tuned to obtain equivalent impedance fitting with an optimal $f_{\mathrm{AC}}$ in the $\mathrm{MHz}$ range. Indeed, if the sensor structure allows, sensing in the $\mathrm{GHz}$ frequency range is also possible, typically leveraging resonance, even on a monolithic CMOS chip (designed with specific RF/microwave criteria) as demonstrated recently for cell dielectric spectroscopy [82].

4.4. Differential Scheme. Finally, whenever possible, it is advisable to adopt a differential configuration. As illustrated in Figure 9(b), a second impedance $Z 2$ matched with the sensor impedance $Z 1$ needs to be available. The series of the two impedances is driven by a differential signal. Starting from a single-ended signal $V_{\mathrm{AC}}$, the signal driving $Z 2$ in counterphase can be easily generated with an inverting buffer. $Z 2$ can be either fixed, that is, equal to the value of $Z 1$ in the initial rest condition, or changing in counterphase with respect to $Z 1$ (i.e., when $Z 1$ increases by a positive amount $\Delta Z, Z 2$ must simultaneously decrease by the same amount). The latter case offers the maximum sensitivity since the difference signal is doubled. When the sensors are perfectly balanced, no current flows in the TIA, giving a zero output signal. In the presence of an event altering the sensor, the two impedances become unbalanced, and the difference current $\Delta I$ is amplified by the TIA. Consequently, despite a doubling of the sensor size, the important advantages of the differential configuration are as follows:

(1) Significant improvement of the DR, since only the difference current $\Delta I$ (produced by $\Delta Z$ ) is amplified and not the total $I$ (given by the total $Z$ ).

(2) Rejection of common-mode noise and disturbances picked up by both $Z 1$ and $Z 2$.

(3) Rejection of environmental effects, such as thermal drifts, affecting both sensors.

(4) Rejection of the noise of the $V_{\mathrm{AC}}$ source [87].

From the point of view of noise, this solution is critical for two reasons. First, the addition of the inverting buffer should not introduce additional noise, thus requiring the noise of the buffer, when referred to the circuit input, to be negligible with respect to the front-end noise contribution. Furthermore, since a second identical impedance is connected to the virtual ground, the sensor capacitance doubles, with possibly relevant impact on the noise, as pointed out above.

Finally, it is worth noting that $Z 2$ can be either a physical replica of the sensor or a dummy impedance equal to the sensor equivalent impedance $Z 1$. Interestingly, the dummy impedance $Z 2$ can be automatically tuned in magnitude and phase to accurately match $Z 1$ even in the presence of parasitic terms [87].

\section{Conclusions}

The results here reviewed have shown the possibility of leveraging high-resolution impedance sensing to detect micronsized objects and nanometric displacement. In particular, the state of the art in capacitance sensing down to zeptoFarad resolution has been analyzed. Key design guidelines, including electrodes and electronics codesign, monolithic integration, parasitics minimization, SNR maximization, and differential strategies, have been extracted from the examples and briefly discussed.

Despite its great versatility, impedance has a weak aspect: it is not specific. In particular, temperature variations affect the impedance of materials. Thus, when measuring a tiny $\Delta Z$, it is important to keep under control all the environmental parameters and cross-sensitivity effects. Luckily, environmental drifts are commonly very slow with respect to the 
detection time scales, so that they can be easily discriminated and filtered. In any case, when measuring a variation $\Delta Z / Z$ in the ppm range (i.e., DR $>120 \mathrm{~dB}$ ), all the components of the sensor conditioning chain must grant a ppm stability.

On the digital processing side, not discussed in this review focusing on devices and analog electronics, several important functions are being progressively embedded in the sensor, such as calibration, fault detection, and autodiagnostics. Clearly, the miniaturization of massively produced sensors, beyond their pervasive dissemination in the smart world, enables new sensing paradigms where complementary sensors are combined in a cooperative way (such as MEMS accelerometers and gyroscopes in inertial navigation units [88]) in order to improve their sensing capabilities.

\section{Competing Interests}

The author declares that there are no competing interests regarding the publication of this paper.

\section{Acknowledgments}

The research activities, here reviewed, to which the author had the opportunity to contribute were led by M. Sampietro and G. Ferrari (Politecnico di Milano), to whom the author's infinite gratitude is devoted for constant support. Very valuable collaborations with $\mathrm{A}$. Melloni and F. Morichetti (Politecnico di Milano) for integrated photonics, with J. Voldman (MIT), J. Emnéus, A. Heiskanen, and C. Caviglia (Denmark Technical University) for microfluidics and cell detection, and with T. Albrecth (Imperial College London) for nanopores are gratefully acknowledged.

\section{References}

[1] J. Z. Chen, A. A. Darhuber, S. M. Troian, and S. Wagner, "Capacitive sensing of droplets for microfluidic devices based on thermocapillary actuation," Lab on a Chip, vol. 4, no. 5, pp. 473-480, 2004.

[2] K. Mohri, T. Uchiyama, L. V. Panina, M. Yamamoto, and K. Bushida, "Recent advances of amorphous wire CMOS IC magneto-impedance sensors: innovative high-performance micromagnetic sensor chip," Journal of Sensors, vol. 2015, Article ID 718069, 8 pages, 2015.

[3] G. Gervasoni, M. Carminati, G. Ferrari et al., "A 12-channel dual-lock-in platform for magneto-resistive DNA detection with ppm resolution," in Proceedings of the 10th IEEE Biomedical Circuits and Systems Conference (BioCAS '14), pp. 316-319, October 2014.

[4] M. Demori, V. Ferrari, P. Poesio, and D. Strazza, "A microfluidic capacitance sensor for fluid discrimination and characterization," Sensors and Actuators, A: Physical, vol. 172, no. 1, pp. 212219, 2011.

[5] S. Murali, X. G. Xia, A. V. Jagtiani, J. Carletta, and J. Zhe, "Capacitive Coulter counting: detection of metal wear particles in lubricant using a microfluidic device," Smart Materials and Structures, vol. 18, no. 3, Article ID 037001, 2009.

[6] D. Silvera-Tawil, D. Rye, M. Soleimani, and M. Velonaki, "Electrical impedance tomography for artificial sensitive robotic skin: a review," IEEE Sensors Journal, vol. 15, no. 4, pp. 20012016, 2015.

[7] E. Al Hosani, M. Zhang, and M. Soleimani, "A limited region electrical capacitance tomography for detection of deposits in pipelines," IEEE Sensors Journal, vol. 15, no. 11, pp. 6089-6099, 2015.

[8] A. J. Fleming, "A review of nanometer resolution position sensors: operation and performance," Sensors and Actuators, A: Physical, vol. 190, pp. 106-126, 2013.

[9] L. Fumagalli, G. Ferrari, M. Sampietro, and G. Gomila, "Quantitative nanoscale dielectric microscopy of single-layer supported biomembranes," Nano Letters, vol. 9, no. 4, pp. 1604-1608, 2009.

[10] M. Carminati, G. Ferrari, and M. Sampietro, "Attofarad resolution potentiostat for electrochemical measurements on nanoscale biomolecular interfacial systems," Review of Scientific Instruments, vol. 80, no. 12, 2009.

[11] M. Carminati, L. Pedalà, E. Bianchi et al., "Capacitive detection of micrometric airborne particulate matter for solid-state personal air quality monitors," Sensors and Actuators A: Physical, vol. 219, pp. 80-87, 2014.

[12] J. A. Geen, S. J. Sherman, J. F. Chang, and S. R. Lewis, "Singlechip surface micromachined integrated gyroscope with 50 \%/ h allan deviation," IEEE Journal of Solid-State Circuits, vol. 37, no. 12, pp. 1860-1866, 2002.

[13] P. Ciccarella, M. Carminati, M. Sampietro, and G. Ferrari, "CMOS monolithic airborne particulate matter detector based on 32 capacitive sensors with a resolution of $65 \mathrm{zF}$ rms," IEEE Journal of Solid-State Circuits, vol. 51, no. 11, pp. 2545-2553, 2016.

[14] H.-K. Kim, S. Lee, and K.-S. Yun, "Capacitive tactile sensor array for touch screen application," Sensors and Actuators A: Physical, vol. 165, no. 1, pp. 2-7, 2011.

[15] J.-W. Lee, D.-J. Min, J. Kim, and W. Kim, "A 600-dpi capacitive fingerprint sensor chip and image-synthesis technique," IEEE Journal of Solid-State Circuits, vol. 34, no. 4, pp. 469-475, 1999.

[16] J. Wei, C. Yue, M. van der Velden et al., "Design, fabrication and characterization of a femto-farad capacitive sensor for pico-liter liquid monitoring," Sensors and Actuators A: Physical, vol. 162, no. 2, pp. 406-417, 2010.

[17] C. Elbuken, T. Glawdel, D. Chan, and C. L. Ren, "Detection of microdroplet size and speed using capacitive sensors," Sensors and Actuators, A: Physical, vol. 171, no. 2, pp. 55-62, 2011.

[18] Y. Xu, X. Xie, Y. Duan, L. Wang, Z. Cheng, and J. Cheng, "A review of impedance measurements of whole cells," Biosensors and Bioelectronics, vol. 77, pp. 824-836, 2016.

[19] O. A. Saleh and L. L. Sohn, "Quantitative sensing of nanoscale colloids using a microchip Coulter counter," Review of Scientific Instruments, vol. 72, no. 12, pp. 4449-4451, 2001.

[20] J.-L. Fraikin, T. Teesalu, C. M. McKenney, E. Ruoslahti, and A. N. Cleland, "A high-throughput label-free nanoparticle analyser," Nature Nanotechnology, vol. 6, no. 5, pp. 308-313, 2011.

[21] K. Mathwig, T. Albrecht, E. D. Goluch, and L. Rassaei, "Challenges of biomolecular detection at the nanoscale: nanopores and microelectrodes," Analytical Chemistry, vol. 87, no. 11, pp. 5470-5475, 2015.

[22] N. Haandbæk, O. With, S. C. Bürgel, F. Heer, and A. Hierlemann, "Resonance-enhanced microfluidic impedance cytometer for detection of single bacteria," Lab on a Chip, vol. 14, no. 17, pp. 3313-3324, 2014.

[23] M. Carminati, M. Giacometti, M. Sampietro, S. Chiodini, T. Doles, and G. Ferrari, "Parallelizable microfluidic resistive online detector of micrometric aggregates of biopharmaceutical antibodies," Procedia Engineering, vol. 168, pp. 1438-1441, 2016. 
[24] Y. Song, H. Zhang, C. H. Chon, S. Chen, X. Pan, and D. Li, "Counting bacteria on a microfluidic chip," Analytica Chimica Acta, vol. 681, no. 1-2, pp. 82-86, 2010.

[25] Y. Song, H. Zhang, C. H. Chon, X. Pan, and D. Li, "Nanoparticle detection by microfluidic Resistive Pulse Sensor with a submicron sensing gate and dual detecting channels-two stage differential amplifier," Sensors and Actuators, B: Chemical, vol. 155, no. 2, pp. 930-936, 2011.

[26] K. R. Balakrishnan, G. Anwar, M. R. Chapman, T. Nguyen, A. Kesavaraju, and L. L. Sohn, "Node-pore sensing: a robust, highdynamic range method for detecting biological species," Lab on a Chip, vol. 13, no. 7, pp. 1302-1307, 2013.

[27] G. Gurun, P. Hasler, and F. L. Degertekin, "Front-end receiver electronics for high-frequency monolithic CMUT-on-CMOS imaging arrays," IEEE Transactions on Ultrasonics, Ferroelectrics, and Frequency Control, vol. 58, no. 8, pp. 1658-1668, 2011.

[28] H. Külah, J. Chae, N. Yazdi, and K. Najafi, "Noise analysis and characterization of a sigma-delta capacitive microaccelerometer," IEEE Journal of Solid-State Circuits, vol. 41, no. 2, pp. 352361, 2006.

[29] H. Cai, Y. Wang, Y. Yu et al., "Resistive-pulse measurements with nanopipettes: detection of vascular endothelial growth factor C (VEGF-C) using antibody-decorated nanoparticles," Analytical Chemistry, vol. 87, no. 12, pp. 6403-6410, 2015.

[30] R. L. Fraccari, P. Ciccarella, A. Bahrami, M. Carminati, G. Ferrari, and T. Albrecht, "High-speed detection of DNA translocation in nanopipettes," Nanoscale, vol. 8, no. 14, pp. 7604-7611, 2016.

[31] R. Rodriguez-Trujillo, M. A. Ajine, A. Orzan et al., "Label-free protein detection using a microfluidic Coulter-counter device," Sensors and Actuators B: Chemical, vol. 190, pp. 922-927, 2014.

[32] Y. Han, H. Wu, F. Liu, G. Cheng, and J. Zhe, "Label-free biomarker assay in a microresistive pulse sensor via immunoaggregation," Analytical Chemistry, vol. 86, no. 19, pp. 9717-9722, 2014.

[33] T. Takakura, I. Yanagi, Y. Goto, Y. Ishige, and Y. Kohara, "Single-molecule detection of proteins with antigen-antibody interaction using resistive-pulse sensing of submicron latex particles," Applied Physics Letters, vol. 108, no. 12, Article ID 123701, 2016.

[34] F. Liu, P. Kc, G. Zhang, and J. Zhe, "Microfluidic magnetic bead assay for cell detection," Analytical Chemistry, vol. 88, no. 1, pp. 711-717, 2016.

[35] Y. Han, H. Wu, G. Cheng, and J. Zhe, "A two-stage microresistive pulse immunosensor for pathogen detection," Lab on a Chip, vol. 16, no. 4, pp. 773-779, 2016.

[36] R. Rodriguez-Trujillo, O. Castillo-Fernandez, M. Garrido, M. Arundell, A. Valencia, and G. Gomila, "High-speed particle detection in a micro-Coulter counter with two-dimensional adjustable aperture," Biosensors and Bioelectronics, vol. 24, no. 2, pp. 290-296, 2008.

[37] T. Sun and H. Morgan, "Single-cell microfluidic impedance cytometry: a review," Microfluidics and Nanofluidics, vol. 8, no. 4, pp. 423-443, 2010.

[38] S. Gawad, L. Schild, and Ph. Renaud, "Micromachined impedance spectroscopy flow cytometer for cell analysis and particle sizing," Lab on a Chip, vol. 1, pp. 76-82, 2001.

[39] D. Holmes, D. Pettigrew, C. H. Reccius et al., "Leukocyte analysis and differentiation using high speed microfluidic single cell impedance cytometry," Lab on a Chip, vol. 9, no. 20, pp. 2881-2889, 2009.
[40] C.-M. Lo, C. R. Keese, and I. Giaever, "Impedance analysis of MDCK cells measured by electric cell-substrate impedance sensing," Biophysical Journal, vol. 69, no. 6, pp. 2800-2807, 1995.

[41] M. Vergani, M. Carminati, G. Ferrari et al., "Multichannel bipotentiostat integrated with a microfluidic platform for electrochemical real-time monitoring of cell cultures," IEEE Transactions on Biomedical Circuits and Systems, vol. 6, no. 5, pp. 498-507, 2012.

[42] M. Carminati, C. Caviglia, A. Heiskanen et al., "Theoretical and experimental comparison of microelectrode sensing configurations for impedimetric cell monitoring," in Lecture Notes on Impedance Spectroscopy, vol. 4, pp. 75-82, CRC Press, 2013.

[43] C. Caviglia, K. Zór, L. Montini et al., "Impedimetric toxicity assay in microfluidics using free and liposome encapsulated anti-cancer drugs," Analytical Chemistry, vol. 87, pp. 2204-2212, 2015.

[44] F. Caselli and P. Bisegna, "A simple and robust event-detection algorithm for single-cell impedance cytometry," IEEE Transactions on Biomedical Engineering, vol. 63, no. 2, pp. 415-422, 2016.

[45] P. Xie, X. Cao, Z. Lin, N. Talukder, S. Emaminejad, and M. Javanmard, "Processing gain and noise in multi-electrode impedance cytometers: comprehensive electrical design methodology and characterization," Sensors and Actuators B: Chemical, vol. 241, pp. 672-680, 2017.

[46] C. H. Clausen, G. E. Skands, C. V. Bertelsen, and W. E. Svendsen, "Coplanar electrode layout optimized for increased sensitivity for electrical impedance spectroscopy," Micromachines, vol. 6, no. 1, pp. 110-120, 2015.

[47] M. Carminati, M. D. Vahey, A. Rottigni, G. Ferrari, J. Voldman, and M. Sampietro, "Enhancement of a label-free dielectrophoretic cell sorter with an integrated impedance detection system," in Proceedings of the 14th International Conference on Miniaturized Systems for Chemistry and Life Sciences (MicroTAS '10), pp. 1394-1396, October 2010.

[48] N.-C. Chen, C.-H. Chen, M.-K. Chen, L.-S. Jang, and M.-H. Wang, "Single-cell trapping and impedance measurement utilizing dielectrophoresis in a parallel-plate microfluidic device," Sensors and Actuators B: Chemical, vol. 190, pp. 570-577, 2014.

[49] M. Shaker, L. Colella, F. Caselli, P. Bisegna, and P. Renaud, "An impedance-based flow microcytometer for single cell morphology discrimination," Lab on a Chip, vol. 14, pp. 2548-2555, 2014.

[50] N. Couniot, L. A. Francis, and D. Flandre, "Resonant dielectrophoresis and electrohydrodynamics for high-sensitivity impedance detection of whole-cell bacteria," Lab on a Chip, vol. 15, pp. 3183-3191, 2015.

[51] M. Solsona, W. Olthuis, and A. van den Berg, "Gradient capacitance for solid particle position detection in electrolyte," Procedia Engineering, vol. 168, pp. 1446-1449, 2016.

[52] A. Meir and B. Rubinsky, "Electrical impedance tomographic imaging of a single cell electroporation," Biomedical Microdevices, vol. 16, no. 3, pp. 427-437, 2014.

[53] S. Afshar, E. Salimi, K. Braasch, M. Butler, D. J. Thomson, and G. E. Bridges, "Multi-frequency DEP cytometer employing a microwave sensor for dielectric analysis of single cells," IEEE Transactions on Microwave Theory and Techniques, vol. 64, no. 3, pp. 991-998, 2016.

[54] Y. Park, J.-J. Cha, S. Seo et al., "Ex vivo characterization of ageassociated impedance changes of single vascular endothelial cells using micro electrical impedance spectroscopy with a cell trap," Biomicrofluidics, vol. 10, Article ID 014114, 2016. 
[55] J. Schoendube, D. Wright, R. Zengerle, and P. Koltay, "Singlecell printing based on impedance detection," Biomicrofluidics, vol. 9, no. 1, Article ID 014117, 2015.

[56] J. Guo, X. Huang, and Y. Ai, "On-demand lensless single cell imaging activated by differential resistive pulse sensing," Analytical Chemistry, vol. 87, no. 13, pp. 6516-6519, 2015.

[57] R. Liu, N. Wang, F. Kamili, and A. F. Sarioglu, "Microfluidic CODES: a scalable multiplexedelectronic sensor for orthogonal detection ofparticles in microfluidic channels," Lab on a Chip, vol. 16, no. 8, pp. 1350-1357, 2016.

[58] A. Susloparova, D. Koppenhöfer, J. K. Y. Law, X. T. Vu, and S. Ingebrandt, "Electrical cell-substrate impedance sensing with field-effect transistors is able to unravel cellular adhesion and detachment processes on a single cell level," Lab on a Chip, vol. 15, no. 3, pp. 668-679, 2015.

[59] J. K. Y. Law, A. Susloparova, X. T. Vu et al., "Human T cells monitored by impedance spectrometry using field-effect transistor arrays: a novel tool for single-cell adhesion and migration studies," Biosensors and Bioelectronics, vol. 67, pp. 170-176, 2015.

[60] F. Morichetti, S. Grillanda, M. Carminati et al., "Non-invasive on-chip light observation by contactless waveguide conductivity monitoring," IEEE Journal on Selected Topics in Quantum Electronics, vol. 20, no. 4, 2014.

[61] D. Melati, M. Carminati, S. Grillanda et al., "Contactless integrated photonic probe for light monitoring in indium phosphide-based devices," IET Optoelectronics, vol. 9, no. 4, pp. 146150, 2015.

[62] P. Ciccarella, M. Carminati, G. Ferrari et al., "Impedance sensing CMOS chip for non-invasive light detection in integrated photonics," IEEE Transactions on Circuits and Systems II: Express Briefs, vol. 63, no. 10, pp. 929-933, 2016.

[63] A. Annoni, E. Guglielmi, M. Carminati et al., "Automated routing and control of silicon photonic switch fabrics," IEEE Journal of Selected Topics in Quantum Electronics, vol. 22, no. 6, 2016.

[64] S. Grillanda, M. Carminati, F. Morichetti et al., "Non-invasive monitoring and control in silicon photonics using CMOS integrated electronics," Optica, vol. 1, no. 3, pp. 129-136, 2014.

[65] Y. Li and A. W. Poon, "Actively stabilized silicon microrings with integrated surface-state-absorption photodetectors using a slope-detection method," Optics Express, vol. 24, no. 19, pp. 21286-21300, 2016.

[66] K. Padmaraju and K. Bergman, "Resolving the thermal challenges for silicon microring resonator devices," Nanophotonics, vol. 3, no. 4-5, pp. 269-281, 2014.

[67] B. G. Lee, A. V. Rylyakov, W. M. J. Green et al., "Monolithic silicon integration of scaled photonic switch fabrics, CMOS logic, and device driver circuits," Journal of Lightwave Technology, vol. 32, no. 4, pp. 743-751, 2014.

[68] S. Han, T. J. Seok, N. Quack, B.-W. Yoo, and M. C. Wu, "Large-scale silicon photonic switches with movable directional couplers," Optica, vol. 2, no. 4, pp. 370-375, 2015.

[69] F. Morichetti, S. Zanotto, A. Blancato et al., "Electrochemical optical actuators: controlling the light through ions," in Proceedings of the 18th International Conference on Transparent Optical Networks (ICTON '16), July 2016.

[70] K. Chen, H.-S. Lee, and C. G. Sodini, "A column-row-parallel ASIC architecture for 3-D portable medical ultrasonic imaging," IEEE Journal of Solid-State Circuits, vol. 51, no. 3, pp. 738-751, 2016.
[71] M. Carminati, G. Ferrari, and M. Sampietro, "Emerging miniaturized technologies for airborne particulate matter pervasive monitoring," Measurement, vol. 101, pp. 250-256, 2015.

[72] J. Zhao, M. Liu, L. Liang, W. Wang, and J. Xie, "Airborne particulate matter classification and concentration detection based on 3D printed virtual impactor and quartz crystal microbalance sensor," Sensors and Actuators, A: Physical, vol. 238, pp. 379-388, 2016.

[73] V. Viswam, J. Dragas, A. Shadmani et al., "22.8 Multi-functional microelectrode array system featuring 59,760 electrodes, 2048 electrophysiology channels, impedance and neurotransmitter measurement units," in Proceedings of the IEEE International Solid-State Circuits Conference (ISSCC '16), pp. 394-395, San Francisco, Calif, USA, January 2016.

[74] T. Sun, D. Holmes, S. Gawad, N. G. Green, and H. Morgan, "High speed multi-frequency impedance analysis of single particles in a microfluidic cytometer using maximum length sequences," Lab on a Chip, vol. 7, no. 8, pp. 1034-1040, 2007.

[75] M. Carminati, G. Ferrari, P. Ciccarella et al., "Low-noise instrument for non-invasive monitoring of photonic integrated circuits," in Proceedings of the IEEE International Instrumentation and Measurement Technology Conference (I2MTC '15), pp. 2078-2083, May 2015.

[76] M. Carminati, A. Rottigni, D. Alagna, G. Ferrari, and M. Sampietro, "Compact FPGA-based elaboration platform for wide-bandwidth electrochemical measurements," in Proceedings of the IEEE International Instrumentation and Measurement Technology Conference (I2MTC '12), pp. 264-267, May 2012.

[77] M. Crescentini, M. Bennati, M. Carminati, and M. Tartagni, "Noise limits of CMOS current interfaces for biosensors: a review," IEEE Transactions on Biomedical Circuits and Systems, vol. 8, no. 2, pp. 278-292, 2014.

[78] G. Ferrari, F. Gozzini, A. Molari, and M. Sampietro, “Transimpedance amplifier for high sensitivity current measurements on nanodevices," IEEE Journal of Solid-State Circuits, vol. 44, no. 5, pp. 1609-1616, 2009.

[79] M. Carminati, G. Ferrari, F. Guagliardo, and M. Sampietro, "ZeptoFarad capacitance detection with a miniaturized CMOS current front-end for nanoscale sensors," Sensors and Actuators, A: Physical, vol. 172, no. 1, pp. 117-123, 2011.

[80] D. Bianchi, G. Ferrari, A. Rottigni, and M. Sampietro, "CMOS impedance analyzer for nanosamples investigation operating up to $150 \mathrm{MHz}$ with sub-aF resolution," IEEE Journal of Solid-State Circuits, vol. 49, no. 12, pp. 2748-2757, 2014.

[81] C. Laborde, F. Pittino, H. A. Verhoeven et al., "Real-time imaging of microparticles and living cells with CMOS nanocapacitor arrays," Nature Nanotechnology, vol. 10, no. 9, pp. 791-795, 2015.

[82] J.-C. Chien and A. M. Niknejad, "Oscillator-based reactance sensors with injection locking for high-throughput flow cytometry using microwave dielectric spectroscopy," IEEE Journal of Solid-State Circuits, vol. 51, no. 2, pp. 457-472, 2016.

[83] E. Ghafar-Zadeh, M. Sawan, and D. Therriault, "A $0.18-\mu \mathrm{m}$ CMOS capacitive sensor Lab-on-Chip," Sensors and Actuators, A: Physical, vol. 141, no. 2, pp. 454-462, 2008.

[84] J. K. Rosenstein, M. Wanunu, C. A. Merchant, M. Drndic, and K. L. Shepard, "Integrated nanopore sensing platform with submicrosecond temporal resolution," Nature Methods, vol. 9, no. 5, pp. 487-492, 2012.

[85] D. Bianchi, M. Carminati, G. Ferrari, and M. Sampietro, "CMOS current amplifier for AFM impedance sensing on chip with ZeptoFarad resolution," in Proceedings of the 9th Conference on 
Ph. D. Research in Microelectronics and Electronics (PRIME '13), pp. 61-64, June 2013.

[86] M. Carminati, M. Vergani, G. Ferrari, L. Caranzi, M. Caironi, and M. Sampietro, "Accuracy and resolution limits in quartz and silicon substrates with microelectrodes for electrochemical biosensors," Sensors and Actuators, B: Chemical, vol. 174, pp. 168-175, 2012.

[87] M. Carminati, G. Gervasoni, M. Sampietro, and G. Ferrari, "Note: differential configurations for the mitigation of slow fluctuations limiting the resolution of digital lock-in amplifiers," Review of Scientific Instruments, vol. 87, no. 2, Article ID 026102, 2016.

[88] M. Carminati, G. Ferrari, R. Grassetti, and M. Sampietro, "Realtime data fusion and MEMS sensors fault detection in an aircraft emergency attitude unit based on Kalman filtering," IEEE Sensors Journal, vol. 12, no. 10, pp. 2984-2992, 2012. 


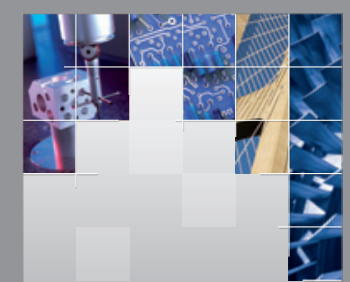

\section{Enfincering}
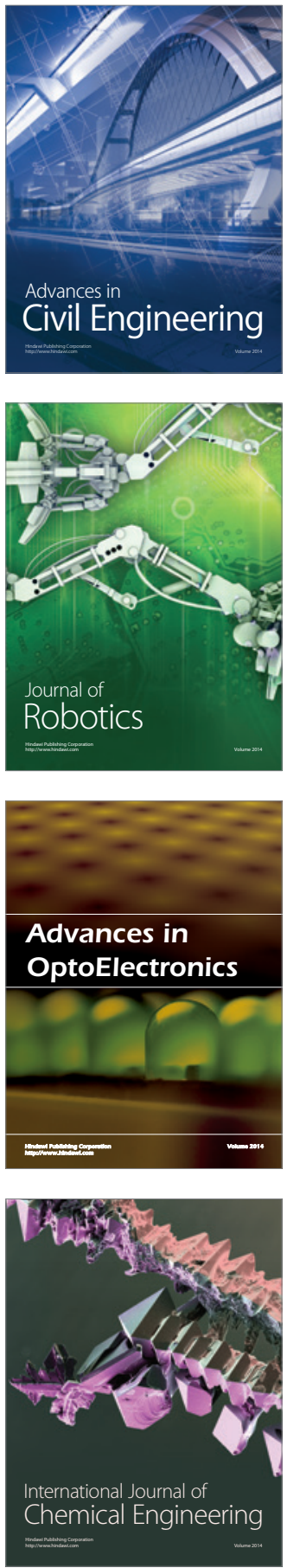

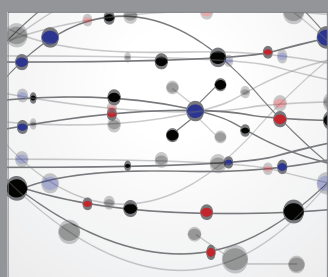

The Scientific World Journal

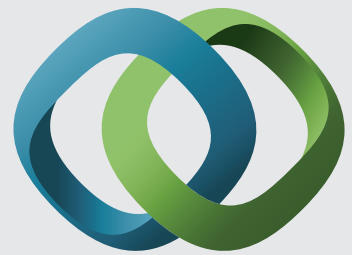

\section{Hindawi}

Submit your manuscripts at

https://www.hindawi.com
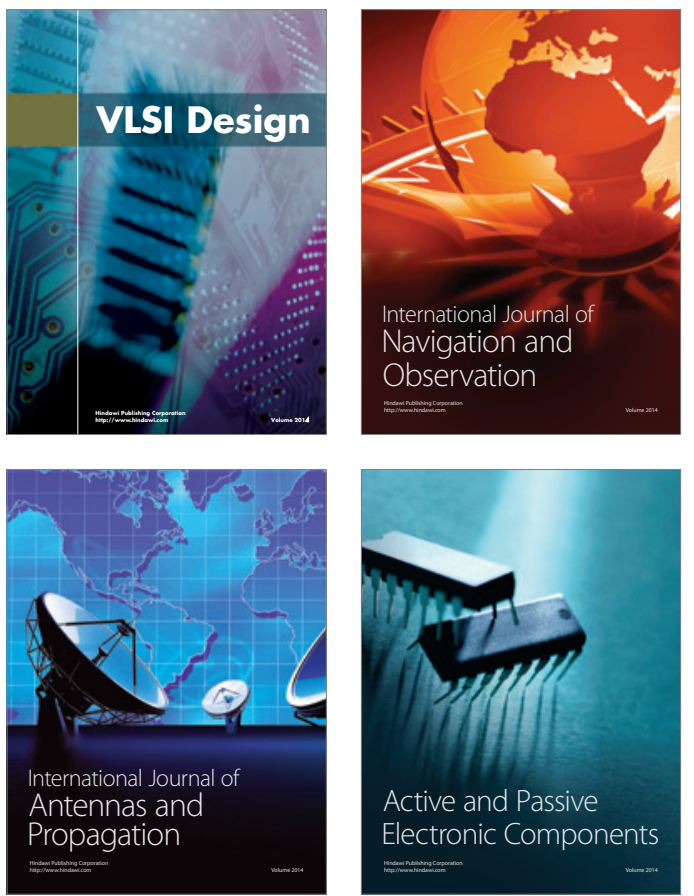
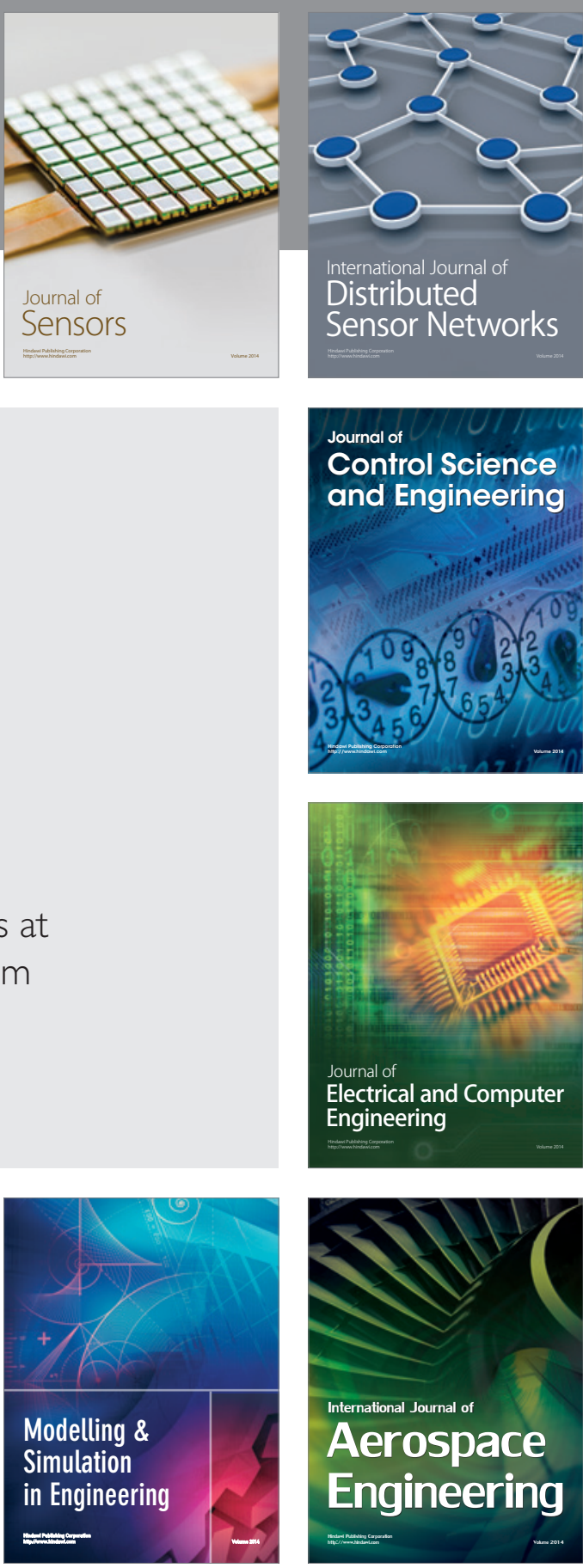

International Journal of

Distributed

Sensor Networks

$-$

Joumal of

Control Science

and Engineering
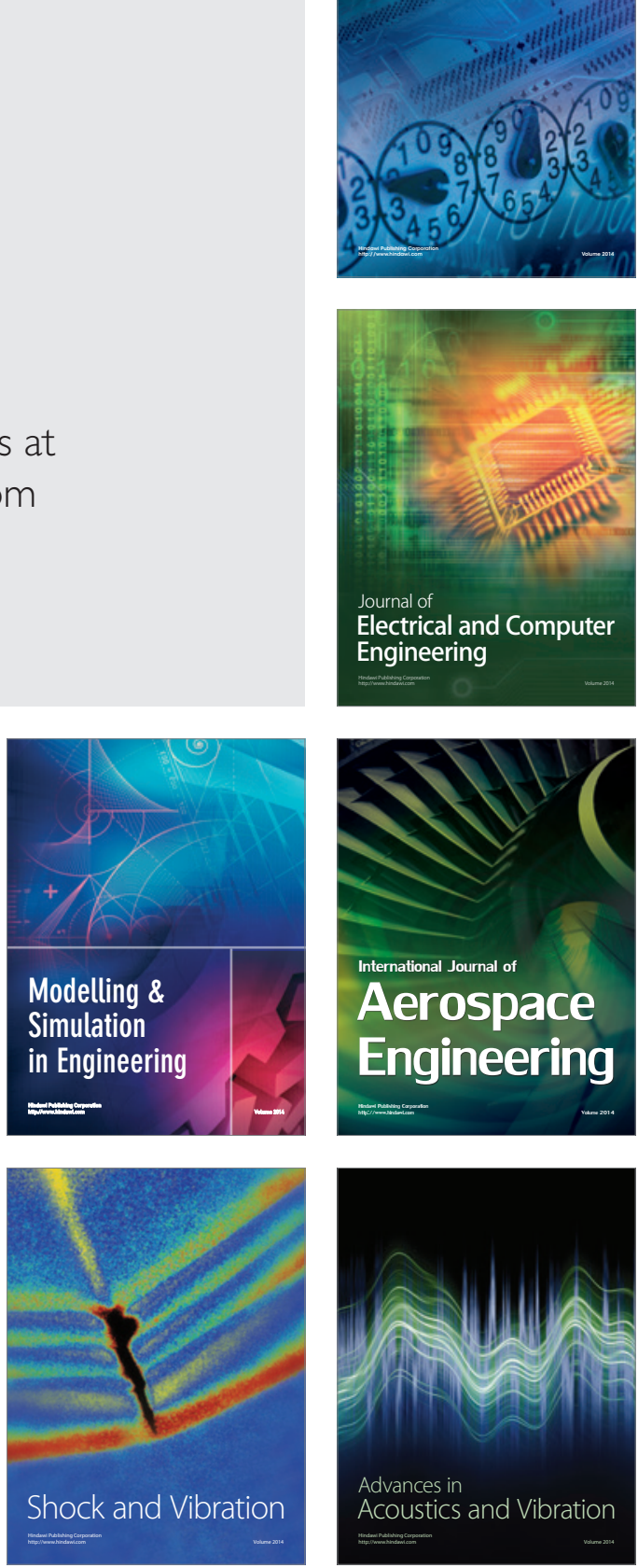